\title{
Knowledge Quartet: dimensões, pesquisas e reflexões sobre o conhecimento profissional do professor que ensina matemática
}

\author{
Knowledge Quartet: dimensions, researches, and reflections about \\ mathematics teacher's professional knowledge
}

\author{
Bárbara Silva Gumiero* \\ ORCID iD 0000-0001-9818-3105 \\ Vinícius Pazuch** \\ ORCID iD 0000-0001-6997-1110
}

\begin{abstract}
Resumo
O Knowledge Quartet (KQ) é uma ferramenta teórica oriunda da prática do professor, composta por quatro dimensões. Neste artigo, apresenta-se uma síntese de literatura, envolvendo 16 artigos selecionados a partir de consultas nas bases de dados Scielo, ERIC, PsycINFO, Web of Science e MathEduc. Discute-se três questões norteadoras: Como têm sido realizadas as pesquisas que têm como foco o KQ? Que dimensões e códigos do KQ são utilizados em pesquisas em Educação Matemática? Como o KQ contribui para a constituição do conhecimento profissional do professor que ensina Matemática? Essas pesquisas destacam as potencialidades do KQ por meio da abordagem qualitativa e do uso de vídeo. Contemplar todas as dimensões e os códigos tem sido a prática mais comum, com ênfase para a dimensão contingência. Identificou-se que o KQ oportuniza reflexão e ampliação do conhecimento docente de forma individual ou colaborativa, valorizando a prática do professor.
\end{abstract}

Palavras-chave: Síntese de Literatura. Educação Matemática. Prática do Professor.

\begin{abstract}
The Knowledge Quartet (KQ) is a theoretical resource of teacher practice, consisting of four dimensions. In this article, we present a literature review of 16 articles selected from queries in the Scielo, ERIC, PsycINFO, Web of Science, and MathEduc databases. Three guiding questions are discussed: How have researches about the KQ been conducted? What are the KQ dimensions and codes used in Mathematics Education researches? How does $\mathrm{KQ}$ contribute to the professional knowledge constitution of a teacher who teaches mathematics? These researches highlight the potential of KQ through the qualitative approach and use of videos. Contemplating all dimensions and codes has been the most common practice, with emphasis on the contingency dimension. It was identified that the KQ allows reflection and development of teacher knowledge in an individual or collaborative way, valuing the teacher's practice.
\end{abstract}

Keywords: Literature review. Mathematics Education. Teacher's practice.

\footnotetext{
* Mestre em Ensino e História das Ciências e da Matemática pela Universidade Federal do ABC (UFABC). Endereço para correspondência: Rua Afonso Furtado de Castro, 163, Jardim Santo André, São Paulo, São Paulo, Brasil, CEP: 08390-140. E-mail: barbara.gumiero@ufabc.edu.br.

** Doutor em Ensino de Ciências e Matemática pela Universidade Luterana do Brasil (ULBRA). Professor Adjunto do Centro de Matemática, Computação e Cognição da Universidade Federal do ABC (UFABC), Santo André, São Paulo, Brasil. Endereço para correspondência: Avenida dos Estados, 5001, Bangú, Santo André, São Paulo, Brasil CEP: 09210-580. E-mail: vinicius.pazuch@ufabc.edu.br.
} 


\section{Introdução}

Dentre os estudos sobre o conhecimento profissional do professor, destacamos as pesquisas de Shulman $(1986,1987)$, que serviram para impulsionar o desenvolvimento de outros estudos. Uma de suas contribuições foi a definição de conhecimento pedagógico do conteúdo como um dos conhecimentos necessários para o professor, caracterizado como "a combinação de conteúdo e pedagogia para compreensão de como tópicos, problemas ou questões são organizados, representados e adaptados para os diversos interesses e aptidões dos alunos, e apresentados para instrução"” (SHULMAN, 1987, p. 8, tradução nossa).

Ball, Thames e Phelps (2008) utilizaram e ampliaram a abordagem de Shulman, estabelecendo domínios de conhecimento matemático para o ensino, conhecido também como Mathematical Knowledge for Teaching (MKT), divididos primeiramente em conhecimento especifico do conteúdo (SMK - sigla em inglês ${ }^{2}$ ) e conhecimento pedagógico do conteúdo (PCK - sigla em inglês ${ }^{3}$ ). A partir do SMK e do PCK, foram estabelecidas subdivisões que contemplavam o conhecimento dos estudantes, do currículo e de como os conteúdos matemáticos se organizam ao longo de cada nível de escolaridade ${ }^{4}$. Entretanto, não é uma tarefa fácil identificar se uma situação pertence a determinado tipo de conhecimento ou de outro, visto que as fronteiras entre essas subdivisões podem se sobrepor. Por exemplo, Coskun e Bostan (2018) questionam se o conhecimento sobre as dificuldades dos estudantes seria classificado em conhecimento do conteúdo e dos estudantes ou em conhecimento do conteúdo e do ensino.

Dessa forma, Rowland, Huckstep e Thwaites (2005) sistematizaram um conjunto de conhecimentos a fim de proporcionar uma reflexão sobre o ensino e sobre o conhecimento do professor a partir de situações da prática docente. Para Ball, Thames e Phelps (2008), o MKT procurava clarificar as noções de Shulman (1986, 1987), adequando-as ao ensino de Matemática, ao realizar uma distinção entre os domínios de conhecimento matemático para o ensino. Em contraste, o conjunto de conhecimentos denominado "Knowledge Quartet" (KQ) tem como foco a classificação das situações em que o conhecimento matemático se aplica ao ensino (ROWLAND, 2013). Além disso, os autores do KQ utilizam o termo conhecimento

\footnotetext{
1 “[...] the blending of content and pedagogy into an understanding of how particular topics, problems, or issues are organized, represented, and adapted to the diverse interests and abilities of learns, ad presented for instruction".

${ }^{2}$ Subject Matter Knowledge.

${ }^{3}$ Pedagogical Content Knowledge.

${ }^{4}$ Todas as subdivisões podem ser encontradas em Ball, Thames e Phelps (2008).
} 
matemático no ensino, já que o conhecimento matemático dos professores é mobilizado durante o ensino e, por isso, seria mais relevante investigar o ambiente da sala de aula.

Diante desses apontamentos, visualizamos que a elaboração do MKT ocorreu a partir do âmbito teórico para a prática, ou seja, foram estabelecidos domínios de conhecimento necessários para a atuação do professor em sala de aula (como um checklist). Por outro lado, o KQ se configurou a partir da prática, observando o trabalho do professor em sala de aula, de forma a evidenciar as situações em que os conhecimentos matemáticos são mobilizados.

Os trabalhos de Shulman representaram um marco nas pesquisas sobre o conhecimento profissional docente durante as décadas de 80 e 90 e se estendem até os dias atuais. Já os estudos de Ball e seus colaboradores foram amplamente difundidos principalmente em 2008: destacaram o desenvolvimento da teoria de Shulman, porém com uma abordagem específica para o ensino de Matemática. O primeiro artigo sobre o KQ foi publicado em 2005, e também tem como foco o ensino de Matemática. Porém, esse conjunto de conhecimento vem ganhando notoriedade desde 2013, quando pesquisas envolvendo o KQ foram desenvolvidas por pesquisadores de diferentes grupos, além dos próprios autores.

$\mathrm{O}$ KQ foi originado de um estudo realizado com estagiários que atuavam com crianças de 3 a 11 anos, em que foram analisados desde o planejamento de suas aulas até a realização destas, filmadas para posterior análise. Ao observar os dados, os pesquisadores identificaram ações significantes relacionadas ao conhecimento específico do conteúdo e também ao conhecimento matemático pedagógico, as quais caracterizaram como códigos. Foram gerados 20 códigos, cada um com um nome específico, que indicava o tipo de ação realizada. Eles foram divididos em quatro categorias ou dimensões - fundamento, transformação, conexão e contingência -, as quais compõem o KQ (ROWLAND, 2013; TURNER; ROWLAND, 2008).

As dimensões e os respectivos códigos que as formam, segundo Rowland (2013), são apresentados no Quadro 1:

\begin{tabular}{|c|l|}
\hline Dimensão & \multicolumn{1}{c|}{ Código } \\
\hline \multirow{5}{*}{ Fundamento } & - Consciência de propósitos \\
& - Identificação de erros \\
& - Conhecimento notório do conteúdo \\
& - Fundamentos subjacentes da pedagogia \\
& - Uso da terminologia \\
& - Uso de livros didáticos \\
& - Dependência dos procedimentos \\
\hline \multirow{5}{*}{ Transformação } & - Demonstrações feitas pelos professores \\
& - Uso de materiais instrucionais \\
& - Escolha de representações \\
& - Escolha de exemplos \\
\hline
\end{tabular}




\begin{tabular}{|c|l|}
\hline & - Conexões entre os processos \\
& - Conexões entre os conceitos \\
& - Antecipação da complexidade \\
& - Decisões sobre sequências \\
& - Reconhecimento da adequação do conceito \\
\hline \multirow{5}{*}{ Contingência } & - Resposta às ideias dos estudantes \\
& - Desvio da agenda planejada \\
& - Insight dos professores \\
& $-($ In)disponibilidade de recursos \\
\hline
\end{tabular}

Quadro 1 - Dimensões do KQ e seus respectivos códigos

Fonte: Elaborado pelos autores, com base em ROWLAND (2013)

A partir dos códigos, os autores estabeleceram compreensões sobre as quatro dimensões: (1) fundamento relaciona-se ao conhecimento e à compreensão da Matemática por si mesma, assim como aos propósitos da Educação Matemática e às condições para que os estudantes aprendam Matemática da melhor maneira; (2) transformação refere-se à apresentação das ideias para os estudantes, na forma de analogias, ilustrações, exemplos, explicações e demonstrações; (3) conexão abrange a sequência do material para o ensino e a consciência das necessidades cognitivas relativas a diferentes tópicos e tarefas; e (4) contingência representa a habilidade para responder de maneira convincente e fundamentada aos eventos não planejados no processo de ensino e de aprendizagem (ROWLAND, 2013).

Sendo o KQ uma ferramenta teórica nova e ainda em desenvolvimento, podemos explorar e apresentar as suas potencialidades e seus desafios a partir de pesquisas internacionais e incentivar a elaboração de novos estudos nessa temática. Por ter o KQ sido desenvolvido a partir da prática, ele aparece como uma ferramenta que pode abordar o conteúdo e o ensino de Matemática, a partir de situações da sala de aula, reconhecendo e valorizando "onde" o professor está e o ajudando a prosseguir, a ampliar seus conhecimentos e a superar dificuldades encontradas em momentos de ensino e de aprendizagem em aulas de Matemática (ROWLAND; HUCKSTEP; THWAITES, 2005).

O KQ pode propiciar momentos de reflexão sobre planejamento e execução das aulas com base em sua própria estrutura. Seus autores buscavam por um esquema facilmente compreensível e um pequeno número de categorias conceituais que pudesse ser lembrado e servisse para estruturar discussões entre professores e observadores. (ROWLAND; HUCKSTEP; THWAITES, 2005). Assim, os professores, ao lembrarem do KQ, se questionam e justificam suas decisões, o que leva a um processo reflexivo, como foi discutido no trabalho de Turner (2012).

Considerando a importância das situações da prática e o conhecimento envolvido nelas, esta síntese de literatura se propõe a responder às seguintes questões: Como têm sido realizadas as pesquisas que têm como foco o KQ? Que dimensões e códigos do KQ são 
utilizados em pesquisas em Educação Matemática? Como o KQ contribui para a constituição do conhecimento profissional do professor que ensina Matemática?

O material que serviu de base para o trabalho aqui exposto é fruto de consultas em base de dados nacionais e internacionais e os procedimentos metodológicos utilizados serão descritos a seguir. Após, apresentaremos as análises verticais e horizontais, sendo a primeira composta pelos artigos selecionados e a outra elaborada a partir da comparação dos artigos com base em nove aspectos a serem especificados. Por fim, retomaremos as questões de pesquisa, para discutir os resultados e as conclusões e apontar pesquisas futuras.

\section{Procedimentos metodológicos}

Este artigo trata de uma síntese de literatura, a qual pode ser entendida como um método para analisar um expressivo número de informações, mapear áreas de incerteza, identificar "onde" um número reduzido ou nenhuma pesquisa foi realizado e até "onde" são necessárias novas pesquisas. Sínteses também podem fornecer uma orientação para futuros estudos e intervenções, apresentando resumos sobre determinado assunto (PETTICREW; ROBERTS, 2006).

Tomamos como referências as pesquisas de Depaepe, Verschaffel e Kelchtermans (2013) e Stahnke, Schueler e Roesken-Winter (2016), publicadas em revistas bem avaliadas no Qualis CAPES e com alto fator de impacto. Essas pesquisas apresentam revisões sistemáticas de literatura, cujas buscas foram realizadas nas bases: ERIC, PsycINFO e Web of Science (DEPAEPE; VERSCHAFFEL; KELCHTERMANS, 2013) e ERIC, PsycINFO e MathEduc (STAHNKE; SCHUELER; ROESKEN-WINTER, 2016). Assim, para o desenvolvimento desta síntese de literatura, realizamos uma busca entre março e dezembro de 2018 nas bases de dados: Scielo, ERIC, PsycINFO, Web of Science e MathEduc, as quais reúnem um número expressivo das pesquisas educacionais no Brasil e no mundo.

Os descritores foram determinados com a condição de que estivessem presentes no resumo, no título e/ou nas palavras-chave. Além disso, foram escritos em inglês, mesmo em buscas no Scielo, já que as publicações nacionais apresentam título, resumo e palavras-chave em outra língua. Assim, utilizamos como descritores as expressões "Knowledge Quartet" (entre aspas, para garantir que fosse um único termo de pesquisa) e mathematics. A partir desses critérios, encontramos 16 artigos, os quais estão descritos em ordem cronológica no Quadro 2. 


\begin{tabular}{|c|c|c|}
\hline Título & Autor & País \\
\hline $\begin{array}{l}\text { Elementary Teachers' Mathematics Subject Knowledge: } \\
\text { the Knowledge Quartet and the Case of Naomi }\end{array}$ & $\begin{array}{l}\text { Rowland, Huckstep e Thwaites } \\
\text { (2005) }\end{array}$ & $\begin{array}{l}\text { Reino } \\
\text { Unido }\end{array}$ \\
\hline $\begin{array}{l}\text { Developing and Using the 'Knowledge Quartet': a } \\
\text { Framework for the Observation of Mathematics } \\
\text { Teaching }\end{array}$ & Rowland e Turner (2007) & $\begin{array}{l}\text { Reino } \\
\text { Unido }\end{array}$ \\
\hline $\begin{array}{l}\text { Analysing secondary mathematics teaching with the } \\
\text { Knowledge Quartet }\end{array}$ & $\begin{array}{l}\text { Thwaites, Jared e Rowland } \\
\text { (2011) }\end{array}$ & $\begin{array}{l}\text { Reino } \\
\text { Unido }\end{array}$ \\
\hline $\begin{array}{l}\text { Using the Knowledge Quartet to develop mathematics } \\
\text { content knowledge: the role of reflection on professional } \\
\text { development }\end{array}$ & Turner (2012) & $\begin{array}{l}\text { Reino } \\
\text { Unido }\end{array}$ \\
\hline $\begin{array}{l}\text { Perspectives on pre-service teacher knowledge for } \\
\text { teaching early algebra }\end{array}$ & Mcauliffe e Lubben (2013) & $\begin{array}{l}\text { África do } \\
\quad \text { Sul }\end{array}$ \\
\hline $\begin{array}{l}\text { Research into teacher knowledge: a stimulus for } \\
\text { development in mathematics teacher education practice }\end{array}$ & $\begin{array}{l}\text { Rowland, Turner e Thwaites } \\
\text { (2013) }\end{array}$ & $\begin{array}{l}\text { Reino } \\
\text { Unido }\end{array}$ \\
\hline $\begin{array}{l}\text { Using the Knowledge Quartet to quantify mathematical } \\
\text { knowledge in teaching: the development of a protocol } \\
\text { for Initial Teacher Education }\end{array}$ & Weston (2013) & $\begin{array}{l}\text { Estados } \\
\text { Unidos da } \\
\text { América } \\
\end{array}$ \\
\hline $\begin{array}{l}\text { Research-led development of primary school teachers' } \\
\text { mathematical knowledge for teaching: a case study }\end{array}$ & Abdulhamid e Venkat (2014) & $\begin{array}{l}\text { África do } \\
\quad \text { Sul }\end{array}$ \\
\hline $\begin{array}{l}\text { The use of video analysis and the Knowledge Quartet in } \\
\text { mathematics teacher education programmes }\end{array}$ & Liston (2015) & Irlanda \\
\hline Triggers of contingency in mathematics teaching & $\begin{array}{l}\text { Rowland, Thwaites e Jared } \\
\qquad(2015)\end{array}$ & $\begin{array}{l}\text { Reino } \\
\text { Unido }\end{array}$ \\
\hline $\begin{array}{l}\text { Prospective Secondary Mathematics Teachers' } \\
\text { Perspectives and Mathematical Knowledge for Teaching }\end{array}$ & Karagöz-Akar (2016) & Turquia \\
\hline $\begin{array}{l}\text { A case study of pedagogy of mathematics support tutors } \\
\text { without a background in mathematics education }\end{array}$ & Walsh (2016) & Irlanda \\
\hline $\begin{array}{l}\text { The Analysis of a Novice Primary Teachers' } \\
\text { Mathematical Knowledge in Teaching: Area } \\
\text { Measurement }\end{array}$ & Coskun e Bostan (2018) & Turquia \\
\hline $\begin{array}{l}\text { Conceptualizations of professional knowledge for } \\
\text { teachers of mathematics }\end{array}$ & Neubrand (2018) & Alemanha \\
\hline $\begin{array}{l}\text { The Knowledge Quartet in the Light of the Literature on } \\
\text { Subject Matter and Pedagogical Content Knowledge }\end{array}$ & Ünver (2018a) & Turquia \\
\hline $\begin{array}{l}\text { Views of Mathematics Student Teachers on Teacher } \\
\text { Insights into the Teaching Process }\end{array}$ & Ünver (2018b) & Turquia \\
\hline
\end{tabular}

Quadro 2 - Artigos selecionados

Fonte: Elaborado pelos autores (2019)

Com relação às bases de dados, encontramos onze artigos em ERIC, sete em MathEduc, sete em Web of Science, dois em PsycINFO e nenhum no Scielo. Nove desses artigos foram encontrados em mais de uma base de dados.

A estrutura desta síntese de literatura foi composta por duas etapas: uma análise 
vertical e uma análise horizontal. Com base no artigo de Depaepe, Verschaffel e Kelchtermans (2013)

Primeiramente, nós realizamos uma análise vertical [...] de cada um dos 60 artigos de pesquisa que foram incluídos em nosso conjunto de dados. Nesta análise vertical, a unidade de análise foi o artigo. Cada artigo foi resumido em um esquema de classificação incluindo nove aspectos ${ }^{5}$ (DEPAEPE; VERSCHAFFEL; KELCHTERMANS, 2013, p. 15, tradução nossa).

$\mathrm{Na}$ síntese de literatura, as análises verticais dos 16 artigos também contemplaram nove aspectos: (1) foco da investigação - o objetivo do artigo e/ou questão de pesquisa, (2) procedimentos metodológicos, (3) domínio matemático, (4) país de desenvolvimento da pesquisa, (5) formação de professores, (6) nível de ensino, (7) resultados e conclusões, (8) dimensões e (9) códigos do KQ. Os sete primeiros aspectos contemplam a primeira questão de pesquisa. Já os dois últimos se relacionam com a segunda questão. Para a terceira questão, todos os aspectos são considerados.

Diante desses resultados, realizamos uma análise horizontal "em que a unidade de análise passou de um artigo de pesquisa particular para [...] os nove aspectos derivados. Para cada aspecto, nós comparamos todos os artigos do nosso conjunto de dados em busca de semelhanças e diferenças sistemáticas" (DEPAEPE; VERSCHAFFEL; KELCHTERMANS, 2013, p. 15, tradução nossa). Dessa forma, destacamos as principais características das pesquisas e as potencialidades do KQ na pesquisa em Educação Matemática. No primeiro aspecto (foco da investigação), elaboramos três categorias de forma a identificar os artigos com objetivos semelhantes ou com um mesmo foco. Nos demais aspectos não foi necessária a criação de categorias, tendo em vista que esses aspectos já possuem suas próprias divisões. A seguir, apresentamos os resultados das análises.

\section{Apresentação e discussão dos materiais}

Nesta seção, os resultados serão apresentados separadamente, iniciando pela análise vertical, de forma a detalhar o conteúdo dos artigos analisados nesta síntese de literatura.

\footnotetext{
5 "We first conducted a vertical analysis [...] of each of the 60 reserach articles that were included in our dataset. In this vertical analysis, the unit of analysis was the article. Each article was summarized in a classification scheme including nine aspects".

6 "[...] where the unit of analysis shifted from a particular research article to [...] the nine derived aspects. For each aspect we compared all articles in our dataset in search of systematic similarities and differences".
} 


\subsection{Análise vertical}

Rownland, Huckstep e Thwaites (2005) produziram o primeiro trabalho sobre o KQ. Sua pesquisa teve como objetivo desenvolver empiricamente esse conjunto de conhecimentos, o KQ, a fim de que formadores e professores, sejam eles em formação inicial ou não, possam refletir sobre o ensino e o conhecimento do professor no contexto escolar. O contexto desse estudo foi um curso de um ano de Pós-Graduação em Educação para professores dos Anos Iniciais do Ensino Fundamental no Reino Unido, no qual foram observadas e filmadas 24 aulas. Após a aula, os pesquisadores escreviam sua sinopse descritiva, a partir de suas anotações de campo ou do próprio vídeo. Além disso, os pesquisadores também recebiam uma cópia do planejamento de cada aula.

Como seu objetivo era desenvolver uma teoria, eles identificaram nos episódios das aulas algumas ações significativas, gerando 18 códigos. Então, assistiram novamente às filmagens e elaboraram uma explicação analítica de cada sinopse descritiva, identificando e codificando cada episódio significativo. Os autores apresentaram as quatro dimensões que compõem o KQ e seus respectivos códigos e utilizaram o caso de Naomi, uma professora participante do curso, e suas aulas sobre subtração, para ilustrar como o KQ pode ser utilizado pelos pesquisadores e pela professora. Os autores afirmaram que o KQ ainda estava em desenvolvimento, mas muitos professores o aprovaram e o consideraram como uma ferramenta importante para a reflexão das aulas e da atuação docente.

No mesmo contexto que o artigo anterior e com objetivo semelhante, Rownland e Turner (2007) apresentaram mais um caso no desenvolvimento do KQ com foco nos momentos de reflexão após as aulas. Seguiu-se uma metodologia equivalente à do estudo anterior, diferenciando-se apenas por considerar 17 códigos (e não 18), visto que os códigos conexões entre os processos e conexões entre os conceitos foram considerados como um único: estabelecer conexões. Esse estudo destacou a professora Chloë, que também lecionava nos Anos Iniciais e trabalhou a subtração através de cálculo mental.

Os dados foram discutidos em consonância com cada uma das dimensões do KQ, e ao final, os autores apresentaram um discussion point, sugerindo questões para uma reunião de reflexão, após a aula, entre os professores participantes e seus mentores no curso de PósGraduação. O KQ se mostrou uma ferramenta importante para auxiliar professores formadores a direcionarem a atenção de seus estudantes para o conteúdo da prática em sala de aula e para as formas como o conhecimento de conteúdo pode influenciar no planejamento, na preparação e na execução das aulas. Além disso, em pesquisas futuras, os autores pretendiam 
abordar essa teoria nos Anos Finais do Ensino Fundamental, como veremos no artigo a seguir.

Thwaites, Jared e Rowland (2011) testaram o KQ em salas de aula dos Anos Finais do Ensino Fundamental, pois as pesquisas anteriores envolviam apenas os Anos Iniciais. Para tanto, três professores voluntários, em formação inicial no Reino Unido, foram convidados a ministrar duas aulas. Os pesquisadores observaram e filmaram cada aula e, em uma análise preliminar, selecionaram alguns episódios, os quais serviram como subsídios para uma entrevista de lembrança estimulada com cada um dos professores. Essas entrevistas foram gravadas em áudio, transcritas e analisadas, a fim de identificar ou não os códigos do KQ. Nesse artigo foi apresentado apenas o caso de John, o qual ministrou uma aula sobre equações quadráticas e gráficos de funções. No presente caso, os autores se depararam com novas situações não identificadas nos estudos anteriores, o que os levou a propor dois novos códigos, uso de materiais instrucionais, na dimensão transformação, a partir das explicações do professor sobre uso de gráficos e de como esses devem ser, e (in)disponibilidade de recursos, na dimensão contingência, resultado esse vindo do fato de que o professor havia planejado uma aula com o uso de software, mas não conseguiu utilizá-lo e acabou retornando à lousa.

Turner (2012) discutiu um trabalho com professores dos Anos Iniciais em início de carreira, com objetivo de apontar como esses professores utilizaram o KQ para refletir sobre o conteúdo matemático de seu ensino, com base na seguinte questão de pesquisa: "Como essa reflexão, apoiada pelo KQ, interage com outros fatores para influenciar o desenvolvimento do conhecimento do conteúdo matemático dos professores?"” (TURNER, 2012, p. 254, tradução nossa).

A pesquisa foi desenvolvida com professores que participaram de um estudo durante quatro anos também no Reino Unido. Foi utilizada uma metodologia específica para cada ano da pesquisa, variando a quantidade de aulas filmadas, entrevistas reflexivas pós-aula com lembrança estimulada por vídeo, entrevistas em grupo e produção de relatos reflexivos pelos professores, após assistirem à gravação da própria aula. Além dos códigos do KQ, os dados referentes à reflexão também foram organizados em duas categorias, experiência e trabalho com outros, que seriam os fatores de influência nas reflexões. As três professoras participantes foram Amy, Kate e Jess, as quais trabalharam com contagem, adição e multiplicação/divisão, respectivamente. Para cada professora, o autor caracterizou a forma como a experiência e o

\footnotetext{
7 "How does reflection, supported by the KQ framework, interact with other factors to influence the development of teachers' mathematics content knowledge?".
} 
trabalho com outras pessoas por afetar as reflexões feitas pelas participantes. Todos os professores afirmaram que o KQ os ajudou no desenvolvimento do conhecimento matemático para o ensino. Esse desenvolvimento foi resultado da reflexão sobre a experiência em sala de aula, da experiência de trabalhar com outros e da reflexão sobre as ideias e as práticas dos outros. $\mathrm{O}$ autor sugere que professores experientes podem contribuir com as reflexões dos professores iniciantes, pois a reflexão, por si só, não é capaz de promover o desenvolvimento do conhecimento matemático para o ensino - é necessário um apoio, como o uso do KQ e de relações entre as escolas e as instituições de Ensino Superior.

A pesquisa de McAuliffe e Lubben (2013) teve como objetivo examinar o conhecimento de um professor em formação mobilizado no ensino da early algebra ${ }^{8}$ através da perspectiva do KQ e também do MKT (BALL; THAMES; PHELPS, 2008), a fim de analisar as diferenças entre os resultados encontrados a partir de cada teoria e refletir sobre como essas visões podem colaborar para avançar no conhecimento necessário para o ensino da álgebra.

A pesquisa foi desenvolvida na África do Sul com uma professora dos Anos Iniciais em formação. No trabalho com uma disciplina, a professora planejou e ministrou, para os estudantes do $3^{\circ}$ ano do Ensino Fundamental, uma aula - filmada e analisada - que envolveu early algebra. Os autores destacaram que, a partir do KQ, foi possível identificar e entender que a professora possuía conhecimento sobre funções e sobre a forma de ensinar esse assunto, assim como estava ciente das representações disponíveis para esse ensino. No entanto, as conexões realizadas por ela pareceram rápidas demais, e não permitiram que os estudantes tivessem tempo para refletir. Realizando análises também por meio do MKT, o artigo reconheceu as potencialidades de cada uma das teorias.

Rowland, Turner e Thwaites (2013) tiveram como objetivo explicar algumas mudanças na prática dos formadores de professores, que levaram ao desenvolvimento deles mesmos e do programa de educação de professores em que atuavam. Dessa forma, o texto aborda o que eles aprenderam com as pesquisas, principalmente, no tocante (i) ao papel da teoria na formação inicial, (ii) ao papel das representações e dos exemplos no ensino de Matemática, (iii) ao uso do vídeo na formação inicial de professores e (iv) à estruturação de revisão e reflexão sobre o ensino.

Os pesquisadores notaram que a teoria não é associada à prática dos professores em formação logo em suas primeiras aulas, pois isso se desenvolve a partir das próprias

\footnotetext{
${ }^{8}$ Álgebra trabalhada desde cedo nos Anos Iniciais.
} 
experiências. Nesse sentido, o KQ pode auxiliá-los a criar vínculos entre o que foi aprendido e sua aplicação em sala de aula. Já no uso de exemplos, foi possível compreender melhor as diferentes finalidades para as quais os exemplos são usados e identificar que, em muitas representações, a Matemática não está presente de maneira adequada, sendo necessário um maior cuidado com suas escolhas. Ao utilizarem vídeos durante a formação inicial, os autores levaram seus estudantes a identificar situações bem-sucedidas, e outras nem tanto, que aconteceu com professores iniciantes e a discutir sobre o que poderia ter sido feito, o que levou os estudantes a refletirem sobre a própria prática. Isso também está de acordo com o último tópico, a revisão e reflexão após as aulas, pois são apresentados relatos de professores participantes, como também dos formadores, em que afirmam o quanto eles utilizam o KQ quando estão planejando e refletindo sobre as aulas. Como pesquisadores, os autores aprenderam a direcionar os seus estudantes de forma que refletissem sobre seu ensino e seus conhecimentos matemáticos. Portanto, ao investigarem a prática dos professores em formação, os autores se desenvolveram profissionalmente como educadores de professores, mostrando que tanto o professor como o pesquisador podem aprender um com o outro.

Weston (2013) teve como objetivo desenvolver um protocolo de codificação baseado no KQ que descrevesse quantitativamente o conhecimento matemático no ensino dos professores em formação a partir do programa de Educação Inicial do Professor nos Estados Unidos da América (EUA). Três professores dos Anos Iniciais em formação participaram desse estudo, em que tiveram quatro aulas filmadas e participaram de entrevistas individuais (logo após as aulas) e coletivas. Não houve indicação sobre qual o domínio matemático trabalhado ao longo das aulas. Ao analisar os dados, a autora identificou quais códigos do KQ estavam presentes ou não (apenas dois não foram identificados), se esses eram considerados apropriados (garantiam a todos o acesso à Matemática) ou não (atrapalhavam ou limitavam esse acesso) para aquele momento e, caso fossem apropriados, em que nível ele ocorria (mínimo, intermediário ou máximo).

Também foi feita uma nova classificação para os códigos, a qual permeava todo o processo das quatro aulas ministradas e era dividida em quatro categorias, que variavam de acordo com a quantidade de professores que havia apresentado o código de maneira consistente. Com essa classificação foi possível identificar quais aspectos precisavam ser trabalhados com mais dedicação, ou quais aparentavam ser mais difíceis durante o curso. Dessa forma, também foi possível analisar os dados quantitativamente, agrupando os resultados encontrados em cada nível dentro de cada uma das dimensões do KQ em percentual, o que mostrou que $30 \%$ dos resultados na dimensão conexão estavam no nível 
inadequado, aproximadamente o dobro da quantidade em comparação com as outras dimensões; e possibilitou uma análise dos códigos individualmente em cada dimensão.

Abdulhamid e Venkat (2014) analisaram o desenvolvimento do conhecimento matemático para o ensino em professores dos Anos Iniciais na África do Sul, utilizando o KQ como base para as análises, explorando se essa teoria poderia contribuir no contexto sulafricano. Foi relatado um estudo de caso da professora Sam, a qual participou de um curso de Matemática para o ensino e já atuava há 18 anos. A partir da filmagem, uma aula da professora sobre adição foi transcrita e analisada por meio do KQ e gerou uma entrevista semiestruturada com lembrança estimulada entre os pesquisadores e a professora, tendo como foco as justificativas da professora para as decisões em sala de aula. Foi possível identificar que todas as dimensões estavam presentes, mas nas dimensões transformação e conexão havia algumas lacunas na seleção de exemplos e na progressão relacionada ao conhecimento. Assim, esses pontos foram norteadores da entrevista, na qual foi possível perceber que a professora estava ciente dos seus objetivos na aula, mas não deixou isso explícito no seu raciocínio. Ela reconheceu que nem todos os estudantes entenderam a lição e que deveriam ser adicionadas atividades para melhorar a compreensão dos estudantes. Além disso, a professora reconheceu a importância da reflexão sobre a própria prática para identificar os erros e pensar em formas de resolvê-los. Dessa forma, o KQ mostrou-se útil no contexto da África do Sul, colaborando para o desenvolvimento do conhecimento matemático para professores dos Anos Iniciais e para a identificação das lacunas na formação.

Liston (2015) teve como objetivo evidenciar o potencial da análise de vídeo e do KQ, quando utilizados conjuntamente, para análise e suporte de futuros professores no desenvolvimento do conhecimento matemático para o ensino, tendo como base as seguintes questões de pesquisa: (i) "Qual a eficácia do uso do vídeo e do KQ na análise do SMK e do PCK dos professores em formação e no suporte à sua habilidade de observar o próprio SMK e PCK?"” (LISTON, 2015, p. 2, tradução nossa); (ii) "Quais são as implicações do uso combinado de instrumentos de vídeo e do KQ em programas de formação de professores? ${ }^{10}$ " (LISTON, 2015, p. 2, tradução nossa). Para isso, foi realizado um estudo de caso com uma professora em formação, na Irlanda, a qual teve sua aula de Matemática, em uma turma dos Anos Finais do Ensino Fundamental, filmada para posterior análise e codificação, seguindo os

\footnotetext{
9 "How effective is the use of vídeo and the $K Q$ in analysing pre-service teachers' SMK and PCK and in supporting their ability to observe their own SMK and PCK?".

10 "What are the implications of using the combined instruments of video and the $K Q$ in teacher education programmes?".
} 
códigos do KQ. A aula abordava o teorema de Pitágoras e razões trigonométricas.

A professora também assistiu ao vídeo da própria aula e enviou seus comentários para os formadores, os quais compararam com a análise já realizada, a fim de identificar e discutir quais discrepâncias existiam entre as suas codificações e as da professora. Em relação à primeira questão de pesquisa, a autora concluiu que o uso do vídeo e do KQ apresenta grande potencial para obter uma visão mais profunda do SMK e do PCK. Além disso, a maioria dos códigos utilizados foi semelhante entre a professora e os formadores. Já na segunda questão, o uso dessas ferramentas auxiliaria os professores formadores na compreensão do SMK e do PCK de seus estudantes, podendo direcionar os próprios estudantes, futuros professores, a refletir sobre a própria prática.

O artigo mais recente dos autores Rowland, Thwaites e Jared (2015) traz como ponto central a dimensão contingência e objetiva classificar fatores que desencadeiam momentos contingentes, os quais, segundo os autores, são os estudantes, o professor e as ferramentas e os recursos pedagógicos utilizados. Os dados analisados foram obtidos a partir de filmagens e de entrevistas com os professores participantes em pesquisas anteriores, desde 2002, com professores dos Anos Iniciais e dos Anos Finais do Ensino Fundamental, além de abranger resultados no Reino Unido e em outros países.

O primeiro fator, o estudante, foi responsável pelos momentos de contingência na maioria dos casos, o que geralmente ocorre a partir de um comentário dele. Foram apresentados três subtipos de classificação nessa categoria: (1) resposta do estudante a uma pergunta do professor; (2) resposta espontânea de um estudante a uma atividade ou discussão; e (3) resposta incorreta do estudante. Já o professor reage (1) ignorando, (2) reconhecendo e deixando de lado ou (3) reconhecendo e incorporando essa ideia inesperada do estudante.

O segundo fator discutido foi o próprio professor, quando ele passa por um processo de reflexão em ação, notando que algo precisa ser modificado no planejamento. Por fim, o terceiro fator caracteriza-se pela (in)disponibilidade de ferramentas e recursos, ou seja, recursos que estavam previstos podem se tornar indisponíveis ou alguma ferramenta que não estava presente no planejamento pode ser adotada apropriadamente. Os autores apontaram que muitos eventos contingentes são provocados por mais de um fator, assim como os próprios códigos do $\mathrm{KQ}$ que, em sua maioria, não ocorrem isoladamente. Os autores acreditam que, ao conhecerem esses três fatores que desencadeiam situações de contingência, os professores iniciantes podem reconhecê-los e confrontá-los com mais segurança quando ocorrerem na própria prática. No entanto, novos fatores causadores de momentos contingentes podem aparecer no futuro. 
Karagöz-Akar (2016), para responder a sua questão investigativa - "Como dois futuros professores de matemática dos anos finais do Ensino Fundamental e Ensino Médio, mantendo uma perspectiva de incorporação progressiva em matemática, aprendizagem de matemática e ensino de matemática, revelam seu conhecimento matemático para o ensino em ação? ${ }^{11 " ~(K A R A G O ̈ Z-A K A R, ~ 2016, ~ p . ~ 5, ~ t r a d u c ̧ a ̃ o ~ n o s s a) ~-~ a n a l i s o u ~ p l a n o s ~ d e ~ a u l a, ~}$ filmagem de aulas e artigos de autorreflexão de dois professores, Alex e Sarah, em formação, em uma universidade na Turquia. Ele ministrou aulas sobre bissetriz de um triângulo em uma escola pública, e ela, em uma escola particular, sobre a equação trigonométrica da forma sen $\mathrm{x}$ = a, ambos para o $1^{\mathrm{o}}$ ano do Ensino Médio.

$\mathrm{O}$ autor transcreveu as aulas e as codificou segundo os códigos do KQ. Após isso, comparou com o planejamento e a reflexão feita por cada professor e elaborou narrativas sobre as perspectivas dos futuros professores e o conhecimento matemático para o ensino. Os resultados sugeriram que os dois futuros professores reconhecem que a aprendizagem é um processo ativo e que ambos aceitaram a participação dos estudantes e suas explicações durante as aulas. Para o pesquisador, os participantes utilizaram exemplos adequados e uma sequência gradual para o ensino, considerando o que os estudantes já haviam aprendido e incorporando novos conhecimentos, além de ouvir o raciocínio dos estudantes, identificando lacunas e as utilizando como uma oportunidade para orientá-los da melhor maneira. Portanto, o autor afirma que, ao utilizar o KQ, juntamente com a perspectiva de incorporação progressiva, foi possível revelar o "porquê" por trás das possibilidades e das limitações do conhecimento matemático dos futuros professores, auxiliando os formadores de professores a projetarem ou modificarem métodos e abordagens de ensino.

O artigo de Walsh (2016) teve como finalidade analisar as habilidades pedagógicas de três tutores que não possuíam formação em Educação Matemática, mas participavam de ações de suporte para o ensino de Matemática uma hora por semana em uma universidade na Irlanda. Foram filmadas três aulas de cada tutor. Os resultados foram divididos em duas partes, uma abordando o conhecimento pedagógico geral ${ }^{12}$ (GPK - sigla em inglês) e outra o KQ. Com relação ao GPK, os resultados mostraram que dois tutores precisavam aumentar o tamanho de sua escrita na lousa, todos os tutores tinham que falar mais devagar e melhorar

\footnotetext{
11 "How do two prospective secondary mathematics teachers, holding a progressive incorporation perspective on mathematics, mathematics learning and mathematics teaching, reveal their mathematical knowledge for teaching in action?".

12 "The autor explains GPK as the skills that a teacher needs regardless of the subject that they are teaching. Examples include blackboard work, probing skills when questioning, classroom management, etc." (WALSH, 2016, p. 3)
} 
suas perguntas, pois eram sempre com respostas "sim ou não", além de dar mais tempo para que os estudantes as respondessem. Também foi aconselhado que não fizessem comentários afirmando que as questões eram fáceis ou simples, que dessem espaço para que os estudantes fizessem perguntas e que acompanhassem a compreensão ao longo de todo o processo, e não apenas ao final de uma questão.

Com relação à dimensão fundamento, apenas um tutor teve dificuldade no conteúdo, mas ainda assim todos eles estavam muito presos a resultados de exames e memorização de fórmulas. Em transformação, nem todas as analogias utilizadas favoreceram a compreensão dos estudantes, o que revelou que os tutores deveriam ter selecionado exemplos melhores e apresentado passo a passo o procedimento de cada resolução. Não houve conexão entre os procedimentos matemáticos utilizados e suas aplicações associadas, ainda que a sequência da aula estivesse clara para cada um dos tutores. E, por fim, dois deles responderam bem às ideias do estudante, enquanto o terceiro, devido à sua menor compreensão no assunto, teve mais dificuldade e não se desviou de seu plano de aula, na dimensão contingência. Assim, os autores sugeriram que os departamentos das instituições preparem esses tutores antes de iniciarem as aulas e os acompanhem durante todo o processo, dando um feedback sobre as aulas ministradas.

Coskun e Bostan (2018) tinham como objetivo investigar o conhecimento matemático de um professor iniciante no ensino de medida de área. Assim, desenvolveram um estudo de caso com uma professora iniciante, Ebru, que atuava no $4^{\circ}$ ano em uma escola turca. Para isso, foram realizadas gravações de cinco aulas, entrevistas de recordação estimulada, anotações de campo e conversas informais entre os pesquisadores e a professora após as aulas. As filmagens foram transcritas e codificadas seguindo os códigos do KQ, tendo destaque: uso do livro didático (fundamento), escolha de representações e escolha de exemplos (transformação), conexão entre conceitos (conexão), resposta às ideias dos estudantes e (in) disponibilidade de recursos (contingência).

A participante demonstrou conhecimento matemático adequado nos códigos relacionados à escolha de representações, conexões entre conceitos e uso dos recursos, porém, nos outros três códigos, o seu conhecimento foi considerado não adequado, visto que ela utilizou o livro didático como sua única fonte e de maneira acrítica, os exemplos selecionados não foram desafiadores a ponto de incentivar os estudantes, e acabou por não utilizar um momento contingente como uma oportunidade de discussão em sala de aula. Os pesquisadores afirmaram que as gravações poderiam ser assistidas de maneira colaborativa, a fim de que outros professores analisassem e melhorassem a própria prática a partir da 
perspectiva do outro, tendo consciência dos seus pontos fortes e fracos. Por fim, é também destacado que os formadores de professores precisam reconsiderar o conteúdo das próprias aulas e proporcionar momentos que influenciem a vida docente dos futuros professores. $\mathrm{E}$ podem utilizar o KQ como uma ferramenta de feedback das práticas de seus estudantes.

O artigo de Neubrand (2018) apresenta algumas ideias sobre o conhecimento profissional dos professores de Matemática, a fim de destacar a variedade de concepções e métodos que podem ser encontrados nas pesquisas. Assim, são descritos três projetos (Michigan Project, COACTIV e TEDS-M) e três conceitualizações, dentre as quais está o KQ, que é visto como uma estrutura de observação do conhecimento matemático de professores dos Anos Iniciais, mas que, com o desenvolvimento de novas pesquisas, se ampliou para os outros níveis de ensino. O autor também afirma que o KQ foi capaz de detectar as facetas essenciais de lições discutidas em um fórum, em 2009. Dentre as outras conceitualizações apresentadas, está o MKT, de Ball e seus colaboradores (2008). No entanto, o artigo destaca limitações nas pesquisas sobre conhecimento profissional docente em geral, visto que ainda há uma lacuna entre o conhecimento teórico e a sala de aula. Além disso, os modelos acabam ignorando aspectos afetivos, como a identidade dos professores e o cuidado com os estudantes. Outro ponto destacado é que a elaboração de novas teorias não tem sido suficientemente trabalhada na prática dos professores, tendo em vista o papel social e político que esses profissionais exercem.

Ünver (2018a) procurou examinar indicadores relativos às dimensões do KQ, de acordo com a literatura sobre SMK e PCK, de forma a enfatizar a importância do KQ para a análise desses estudos. Esses indicadores foram elaborados de forma a ampliar o alcance dos códigos em cada uma das dimensões. Um exemplo seria o indicador "mostrar, em seu planejamento, conhecimento de erros comuns e equívocos e tomar medidas para evitá-los", que pode se relacionar com o código identificação de erros na dimensão fundamento e que, segundo o artigo, tem como ideia-chave "errors and misconceptions". Assim, a autora realizou uma revisão de literatura sobre SMK e PCK em estudos que abordavam o KQ, publicados entre 2003 e 2017 . Não há informações sobre como foram realizadas essas buscas.

$\mathrm{Na}$ apresentação dos resultados, foi estruturada uma tabela com todos os indicadores elaborados dentro de cada dimensão e os estudos encontrados que se relacionavam com esses indicadores. Nem todos os indicadores possuíam algum texto relacionado a eles. Os indicadores que aparecem mais frequentemente, de acordo com os resultados, foram errors and misconceptions, representations, make links e children's comments, questions and answers, os quais se referem às dimensões fundamento, transformação, conexão e 
contingência, respectivamente. A autora concluiu que o KQ é uma estrutura para a observação, a análise e o desenvolvimento do ensino de Matemática, além de ser detalhada e abrangente para examinar como o SMK e o PCK dos professores se refletem em seu processo de ensino.

Com o intuito de explorar a opinião dos futuros professores de Matemática em relação aos momentos de insights durante o processo de ensino, Ünver (2018b) desenvolveu um estudo com 30 estudantes que estavam cursando a disciplina Teaching Practice e realizavam tarefas com turmas do Ensino Médio, na Turquia. Dessa forma, o foco foi a dimensão da contingência, abordando especificamente o código "insight dos professores". Para isso, duas perguntas abertas foram elaboradas: "Se seus estudantes não entendem um conteúdo que você está explicando, você pode perceber isso? Como? ${ }^{13}$ " (ÜNVER, 2018b, p. 693, tradução nossa) e "O que você faz quando percebe que seus estudantes não estão entendendo um conteúdo que você está explicando? ${ }^{14 ”}$ (ÜNVER, 2018b, p. 693, tradução nossa). Realizou-se, então, uma análise das respostas dos participantes, gerando categorias.

Com relação à primeira pergunta, apenas um participante declarou não ser capaz de perceber e revelou esperar que os próprios estudantes digam que não entenderam. A maioria dos professores em formação respondeu que perceberia, ao olhar para os estudantes. Outros participantes destacaram que as atitudes dos estudantes, a falta de interesse ou as concepções errôneas também são indicadores de que eles não estão entendendo. Um participante afirmou que essa percepção seria adquirida com a experiência. Já com relação à segunda questão, 16 participantes utilizariam métodos e técnicas diferentes, se percebessem que seus estudantes não estavam entendendo. Diferentes estratégias também foram citadas pelos outros professores, como: ensinar o conteúdo novamente, resolver diferentes exemplos, atrair a atenção, utilizar softwares, ensinar por pares, entre outros. Assim, a autora compreendeu que os insights dos professores seriam moldados pelas ações, pelas características, pelos gestos, pelos discursos e pelas atitudes dos estudantes, concordando ser necessário que os professores em formação adquiram experiência, principalmente no ambiente da sala de aula. Além disso, situações vividas por professores que já atuam poderiam ser discutidas durante os cursos de formação, de maneira que esses futuros professores refletissem sobre como tomar certas atitudes em situações inesperadas.

\footnotetext{
13 "If your students do not understand a subject you are lecturing, can you notice this? How?".

14 "What do you do when you realize that your students do not understand a subject you are lecturing?".
} 


\subsection{Análise horizontal}

Retomando os nove aspectos contemplados na análise vertical, apresentamos os resultados das análises horizontais nos tópicos e nos quadros a seguir.

\subsubsection{Foco da investigação - objetivo do artigo e/ou questão de pesquisa}

Ao observarmos os objetivos ou as questões de pesquisas dos artigos selecionados, identificamos três categorias: (A) Apresentar o KQ e suas potencialidades, cujos trabalhos objetivam demonstrar o desenvolvimento da teoria e suas aplicações em diferentes contextos; (B) Analisar o conhecimento do professor através do KQ e outras teorias, comparando-as, para um resultado mais amplo; e (C) Elaborar uma nova abordagem teórico-metodológica, sendo essa criada a partir do KQ. Os resultados são apresentados no Quadro 3.

\begin{tabular}{|c|c|c|c|}
\hline Artigos & $\mathbf{A}$ & B & $\mathbf{C}$ \\
\hline Rowland, Huckstep e Thwaites (2005) & $\mathrm{x}$ & & \\
\hline Rowland e Turner (2007) & $\mathrm{x}$ & & \\
\hline Thwaites, Jared e Rowland (2011) & $\mathrm{x}$ & & \\
\hline Turner (2012) & $\mathrm{x}$ & & \\
\hline McAuliffe e Lubben (2013) & & $\mathrm{x}$ & \\
\hline Rowland, Turner e Thwaites (2013) & $\mathrm{x}$ & & \\
\hline Weston (2013) & & & $\mathrm{x}$ \\
\hline Abdulhamid e Venkat (2014) & $\mathrm{x}$ & & \\
\hline Liston (2015) & $\mathrm{x}$ & & \\
\hline Rowland, Thwaites e Jared (2015) & $\mathrm{x}$ & & \\
\hline Karagöz-Akar (2016) & & $\mathrm{x}$ & \\
\hline Walsh (2016) & $\mathrm{x}$ & & \\
\hline Coskun e Bostan (2018) & $\mathrm{x}$ & & \\
\hline Neubrand (2018) & & $\mathrm{x}$ & \\
\hline Ünver (2018a) & & & $\mathrm{x}$ \\
\hline Ünver (2018b) & $\mathrm{x}$ & & \\
\hline
\end{tabular}

A partir do Quadro 3 identificamos que a maioria dos artigos possui como objetivo apresentar o KQ como uma ferramenta teórica de análise da prática do professor que ensina Matemática em diferentes níveis de escolaridade, particularmente, em relação aos Anos Iniciais do Ensino Fundamental. Essa apresentação do KQ é relevante na medida em que essa ferramenta teórica está em desenvolvimento. Como destacado anteriormente, a partir de 2013, as pesquisas sobre o KQ começaram a ser realizadas por diferentes pesquisadores, em vários países e com outros objetivos. 


\subsubsection{Procedimentos metodológicos}

Dentre todos os artigos analisados neste trabalho, três aparecem como estudos teóricos (NEUBRAND, 2018; ROWLAND; TURNER; THWAITES, 2013; ÜNVER, 2018a). Além disso, com relação à natureza das pesquisas, somente o trabalho de Weston (2013) se desenvolveu a partir de uma abordagem quantitativa. Assim, devido à predominância da abordagem qualitativa, identificamos a presença de diversos instrumentos de produção de dados que são influenciados por valores, intenções, experiências de vida e condições sociopolíticas, tendo em vista que essa abordagem reconhece o conhecimento como dinâmico e não neutro (ARAÚJO; BORBA, 2004).

\begin{tabular}{|c|c|c|c|c|c|}
\hline Artigos & $\begin{array}{c}\text { Planejamento } \\
\text { das aulas }\end{array}$ & $\begin{array}{c}\text { Filmagem } \\
\text { das aulas }\end{array}$ & Entrevistas & $\begin{array}{c}\text { Produção } \\
\text { de } \\
\text { relatos }^{15}\end{array}$ & $\begin{array}{c}\text { Perguntas } \\
\text { abertas }\end{array}$ \\
\hline Rowland, Huckstep e Thwaites (2005) & $\mathrm{x}$ & $\mathrm{x}$ & & & \\
\hline Rowland e Turner (2007) & $\mathrm{x}$ & $\mathrm{x}$ & & & \\
\hline Thwaites, Jared e Rowland (2011) & & $\mathrm{x}$ & $\mathrm{x}$ & & \\
\hline Turner (2012) & & $\mathrm{x}$ & $\mathrm{x}$ & $\mathrm{x}$ & \\
\hline McAuliffe e Lubben (2013) & $\mathrm{x}$ & $\mathrm{x}$ & & & \\
\hline Weston (2013) & & $\mathrm{x}$ & $\mathrm{x}$ & & \\
\hline Abdulhamid e Venkat (2014) & & $\mathrm{x}$ & $\mathrm{x}$ & & \\
\hline Liston (2015) & & $\mathrm{x}$ & & $\mathrm{x}$ & \\
\hline Rowland, Thwaites e Jared (2015) & & $\mathrm{x}$ & $\mathrm{x}$ & & \\
\hline Karagöz-Akar (2016) & $\mathrm{x}$ & $\mathrm{x}$ & & $\mathrm{x}$ & \\
\hline Walsh (2016) & & $\mathrm{x}$ & & & \\
\hline Coskun e Bostan (2018) & & $\mathrm{x}$ & $\mathrm{x}$ & & \\
\hline Ünver (2018b) & & & & & $\mathrm{x}$ \\
\hline
\end{tabular}

Quadro 4 - Instrumentos de produção de dados Fonte: Elaborado pelos autores (2019).

\subsubsection{Domínio matemático}

Nem todos os artigos que compuseram esta síntese de literatura apresentaram o domínio matemático investigado. Entretanto, a partir dos nove trabalhos que divulgaram essa informação, a maioria abordou a aritmética, o que pode estar relacionado com o nível de ensino em que essas pesquisas foram desenvolvidas, visto que o $\mathrm{KQ}$, inicialmente, foi proposto para os Anos Iniciais do Ensino Fundamental. Porém, destacamos trabalhos que contemplam a álgebra e a geometria, como também o de Karagöz-Akar (2016), o qual contemplou esses dois domínios.

\footnotetext{
${ }^{15}$ Produção escrita feita pelos participantes da pesquisa após a observação ou a execução de aulas, podendo ser um relato, comentário, relatório ou reflexão.
} 


\begin{tabular}{|l|c|c|c|}
\multicolumn{1}{c|}{ Artigos } & Álgebra & Geometria & Aritmética \\
\hline Rowland, Huckstep e Thwaites (2005) & & & X \\
\hline Rowland e Turner (2007) & & & X \\
\hline Thwaites, Jared e Rowland (2011) & X & & X \\
\hline Turner (2012) & & & \\
\hline McAuliffe e Lubben (2013) & X & & X \\
\hline Abdulhamid e Venkat (2014) & & X & \\
\hline Liston (2015) & X & X & \\
\hline Karagöz-Akar (2016) & & \\
\hline Coskun e Bostan (2018) & & \\
\hline
\end{tabular}

\subsubsection{País de desenvolvimento da pesquisa}

Conforme já apresentado no Quadro 2, nenhum dos artigos que fizeram parte dessa análise é de autores brasileiros. Além disso, em todo o continente americano apenas um artigo foi encontrado, tendo como país de desenvolvimento os EUA. A maioria das pesquisas analisadas são do Reino Unido, o que decorre da própria elaboração e desenvolvimento do KQ, já que os autores dessa ferramenta teórica também são os autores desses seis artigos. Destacamos também duas pesquisas no continente africano.

\subsubsection{Formação de professores}

Podemos dividir a formação de professores em dois períodos: Formação Inicial (FI) e Formação Continuada (FC). Observando as análises verticais, vemos um equilíbrio entre o número de trabalhos que contemplam esses períodos; no entanto, nenhum trabalho pode abranger professores em formação e aqueles que já atuam.

\begin{tabular}{|c|c|c|}
\hline Artigos & FI & FC \\
\hline Rowland, Huckstep e Thwaites (2005) & & $\mathrm{X}$ \\
\hline Rowland e Turner (2007) & & $\mathrm{X}$ \\
\hline Thwaites, Jared e Rowland (2011) & $\mathrm{X}$ & \\
\hline Turner (2012) & & $\mathrm{X}$ \\
\hline McAuliffe e Lubben (2013) & $\mathrm{X}$ & \\
\hline Weston (2013) & $\mathrm{X}$ & \\
\hline Abdulhamid e Venkat (2014) & & $\mathrm{x}$ \\
\hline Liston (2015) & $\mathrm{x}$ & \\
\hline Karagöz-Akar (2016) & $\mathrm{X}$ & \\
\hline Coskun e Bostan (2018) & & $\mathrm{X}$ \\
\hline Ünver (2018b) & $\mathrm{X}$ & \\
\hline
\end{tabular}

Quadro 6 - Formação de professores

Fonte: Elaborado pelos autores (2019).

\subsubsection{Nível de ensino}

Como já comentado na subseção sobre domínio matemático, o desenvolvimento do 
KQ se deu através de pesquisas com professores dos Anos Iniciais do Ensino Fundamental (EF), o que, como demonstrado no Quadro 7, fez com que a maioria das pesquisas desta análise também contemplasse esse nível de ensino. Reconhecemos a carência de pesquisas no Ensino Médio e no Ensino Superior.

\begin{tabular}{|c|c|c|c|c|}
\hline Artigos & $\begin{array}{c}\text { EF - Anos } \\
\text { Iniciais }\end{array}$ & $\begin{array}{c}\text { EF - Anos } \\
\text { Finais }\end{array}$ & $\begin{array}{l}\text { Ensino } \\
\text { Médio }\end{array}$ & $\begin{array}{c}\text { Ensino } \\
\text { Superior }\end{array}$ \\
\hline Rowland, Huckstep e Thwaites (2005) & $\mathrm{x}$ & & & \\
\hline Rowland e Turner (2007) & $\mathrm{x}$ & & & \\
\hline Thwaites, Jared e Rowland (2011) & & $\mathrm{x}$ & & \\
\hline Turner (2012) & $\mathrm{x}$ & & & \\
\hline McAuliffe e Lubben (2013) & $\mathrm{x}$ & & & \\
\hline Weston (2013) & $\mathrm{x}$ & & & \\
\hline Abdulhamid e Venkat (2014) & $\mathrm{x}$ & & & \\
\hline Liston (2015) & & $\mathrm{x}$ & & \\
\hline Rowland, Thwaites e Jared (2015) & $\mathrm{x}$ & $\mathrm{x}$ & & \\
\hline Karagöz-Akar (2016) & & & $\mathrm{x}$ & \\
\hline Walsh (2016) & & & & $\mathrm{x}$ \\
\hline Coskun e Bostan (2018) & $\mathrm{x}$ & & & \\
\hline Ünver (2018b) & & & $\mathrm{x}$ & \\
\hline
\end{tabular}

\subsubsection{Resultados e conclusões}

Observando os resultados e as conclusões dessas pesquisas, percebemos que o KQ, mesmo sendo uma ferramenta teórica nova, foi considerado como uma ferramenta importante, tanto para os professores, como para os pesquisadores, já que ambos foram influenciados pelo uso desse, principalmente em momentos de reflexão após as aulas. Além disso, o KQ foi reconhecido e aprovado por professores em contextos diferentes, utilizado juntamente com outras teorias, apresentando resultados mais amplos e se destacando como uma ferramenta que emergiu da prática do professor que ensina Matemática.

\subsubsection{Dimensões do KQ}

Ao analisarmos os artigos com foco nas dimensões do KQ, avaliamos que a maioria dos trabalhos publicados aborda todas as dimensões, mas apenas as pesquisas de Rowland, Thwaites e Jared (2015) e de Ünver (2018b) possuem como objetivo dos seus estudos a dimensão contingência. Dentro do ambiente de sala de aula, momentos contingentes acontecem com frequência, mas não são previsíveis, o que torna difícil o trabalho com esses momentos nos cursos de formação. Porém, como os próprios resultados das pesquisas indicam, o uso de episódios de aulas pode auxiliar na reflexão dos futuros professores sobre 
como agir em situações do tipo.

\subsubsection{Códigos do KQ}

Como consequência dos resultados apresentados no tópico anterior, poucos trabalhos destacam uma escolha restrita de códigos, e 13 dos 16 artigos analisados envolvem todos os códigos do KQ. Os trabalhos de Coskun e Bostan (2018) e Ünver (2018b) deixam explícita a escolha de apenas alguns códigos para o desenvolvimento dos estudos. Já o artigo de Thwaites, Jared e Rowland (2011) inicia abordando todos os códigos, mas, ao final, destaca os códigos uso de materiais instrucionais e (in)disponibilidade de recursos, pois esses dois emergiram dos resultados dessa sua pesquisa.

\begin{tabular}{|c|c|c|c|c|}
\hline Dimensão & Códigos & $\begin{array}{c}\text { Thwaites, Jared } \\
\text { e Rowland } \\
(\mathbf{2 0 1 1 )}\end{array}$ & $\begin{array}{c}\text { Coskun e } \\
\text { Bostan }(2018)\end{array}$ & $\begin{array}{c}\text { Ünver } \\
(2018 b)\end{array}$ \\
\hline Fundamento & Uso de livros didáticos & & $\mathrm{x}$ & \\
\hline \multirow{3}{*}{ Transformação } & Uso de materiais instrucionais & $\mathrm{x}$ & & \\
\hline & Escolha de representações & & $\mathrm{x}$ & \\
\hline & Escolha de exemplos & & $\mathrm{x}$ & \\
\hline Conexão & Conexão entre os conceitos & & $\mathrm{x}$ & \\
\hline \multirow{2}{*}{ Contingência } & Insight dos professores & & & $\mathrm{x}$ \\
\hline & (In)disponibilidade de recursos & $\mathrm{X}$ & $\mathrm{x}$ & \\
\hline
\end{tabular}

Quadro 8 - Códigos em destaque em algumas pesquisas

Fonte: Elaborado pelos autores (2019).

\section{Análise dos resultados: retomada das questões de pesquisa}

Por meio dos resultados sintetizados nas análises verticais e horizontais, retomamos as questões norteadoras do artigo, a fim de respondê-las.

Como têm sido realizadas as pesquisas que têm como foco o $K Q$ ?

As pesquisas cujo foco era o KQ buscavam apresentar essa teoria ainda em desenvolvimento, descrevendo seus impactos e potencialidades em outros países e públicos, além do Reino Unido, onde foi elaborada, sendo esse o país que mais produziu artigos nesse tema. Além disso, as pesquisas se caracterizam, em sua maioria, como qualitativas, empíricas e fortemente influenciadas pelo uso do vídeo nas filmagens das aulas. O KQ aparece como uma ferramenta teórica para reflexão do planejamento e da execução das aulas, possibilitando que professores em formação, já atuantes, e até mesmo formadores de professores aprendam e reflitam sobre as próprias práticas, pois leva em consideração o ambiente escolar e o 
conhecimento matemático no ensino em seus diversos domínios.

Que dimensões e códigos do KQ são utilizados em pesquisas em Educação Matemática?

Após analisarmos os artigos individual e globalmente, identificamos que é comum o uso de todas as dimensões e códigos nos estudos realizados, com o intuito de identificar como ocorre a própria prática. No entanto, a dimensão contingência e seus respectivos códigos, com destaque para in(disponibilidade) de recursos, são evidenciados em algumas pesquisas, ainda que tenhamos trabalhos que selecionem alguns códigos de outras dimensões.

Como o KQ contribui para a constituição do conhecimento profissional do professor que ensina Matemática?

O KQ é uma ferramenta teórica que emergiu do próprio dia a dia do professor, o que o torna mais próximo da sala de aula e do que ocorre no ambiente escolar. Indo além de um checklist, em que conferimos o que aparece ou não, ou o que está certo ou não, o intuito do KQ é possibilitar reflexão e desenvolvimento constante do conhecimento docente, de forma que o próprio professor possa identificar em si mesmo seus avanços e suas limitações.

A partir dos resultados das análises verticais e horizontais, as contribuições do KQ se estendem aos que participam dos processos formativos do professor - licenciados, professores da Educação Básica, pesquisadores - pois pode ser utilizado em cursos de formação inicial ou continuada, em reuniões pedagógicas e por cada professor individualmente. Dessa forma, os resultados da síntese de literatura indicam que o KQ pode contribuir durante as ações do professor, desde o planejamento, passando pelo momento em sala de aula, até a reflexão posterior, valorizando a prática docente e permitindo que os professores produzam e avaliem seus conhecimentos periodicamente.

A partir da discussão dos resultados delimitados pela síntese de literatura, tendo como base as três questões, compilamos as conclusões e as possíveis contribuições para a área de Educação Matemática.

\section{Conclusões e contribuições para a Educação Matemática}

Diante das escolhas metodológicas e da síntese de literatura empreendida, neste artigo, consideramos que o KQ está em construção e que não há produção de autores brasileiros 
sobre a temática. Para tanto, defendemos que a caracterização dessa ferramenta de análise advinda das situações de sala de aula qualifica ainda mais o debate científico sobre o conhecimento profissional do professor que ensina Matemática.

O KQ como uma ferramenta de análise, constituída na e pela reflexão da prática docente, diferencia-se dos estudos sobre o conhecimento profissional docente, que tratam de quais conhecimentos são necessários para o professor ensinar Matemática. Enfatizamos que o estudo das dimensões do KQ como processual permite abordar a evolução dos códigos, ao longo de sua própria constituição.

Dessa maneira, ao analisar situações de sala de aula, novos códigos podem ser revelados, o que demarca a possibilidade de teorizar os conhecimentos mobilizados pelo professor. Além disso, os códigos do KQ podem permitir a identificação de lacunas presentes em processos de formação (inicial ou continuada) de professores que ensinam Matemática. Por fim, consideramos o KQ como potencial ferramenta de análise da prática do professor no sentido de evidenciar o papel social e político que essa profissão exerce na formação do próprio professor e dos estudantes.

Em contraste, compreendemos que a síntese de literatura possui um limite em seus resultados, tendo em vista as escolhas e as decisões tomadas ao longo do processo, sejam pelo uso das bases de dados, pelos descritores ou pela língua. Para além disso, foi possível apresentar o KQ e suas potencialidades, evidenciando algumas pesquisas já consolidadas, incentivando a produção de novos trabalhos principalmente no Brasil, devido à ausência de trabalhos dessa natureza.

Em suma, a ampliação de pesquisas envolvendo o Ensino Médio e o Ensino Superior, englobando também os domínios da álgebra e da geometria se desvelam como possibilidades na área de Educação Matemática. Em particular, pesquisas sobre as dimensões fundamento, transformação e conexão podem contribuir nesse campo de pesquisa, enriquecendo os códigos já consolidados e ampliando e valorizando a discussão sobre o conhecimento profissional do professor que ensina Matemática.

\section{Agradecimentos}

O presente trabalho foi realizado com apoio da Coordenação de Aperfeiçoamento de Pessoal de Nível Superior - Brasil (CAPES) - Código de Financiamento 001. 


\section{Referências}

ABDULHAMID, L.; VENKAT, H. Research-led development of primary school teachers' mathematical knowledge for teaching: a case study. Education as Change, [s.1.], v. 18, n. 1, p. 137150, 2014.

ARAÚJO, J. L.; BORBA, M. C. Construindo pesquisas coletivamente em Educação Matemática. In: BORBA, M. C.; ARAÚJO, J. L. (Org.). Pesquisa qualitativa em Educação Matemática. Belo Horizonte: Autêntica, 2004. p. 25-45.

BALL, D.; THAMES, M. H.; PHELPS, G. Content knowledge for teaching: what makes it special? Journal of Teacher Education, [s.1], v. 59, n. 5, p. 389-407, 2008.

COSKUN, S. D.; BOSTAN, M. I. The analysis of a novice primary teachers' mathematical knowledge in teaching: Area measurement. International Journal for Mathematics Teaching and Learning, [s.1], v. 19, n. 1, p. 1-21, 2018.

DEPAEPE, F.; VERSCHAFFEL, L.; KELCHTERMANS, G. Pedagogical content knowledge: A systematic review of the way in which the concept has pervaded mathematics educational research. Teaching and Teacher Education, [s.1], v. 34, n. 1, p.12-25, 2013.

KARAGÖZ-AKAR, G. Prospective secondary mathematics teachers' perspectives and mathematical knowledge for teaching. Eurasia Journal of Mathematics, Science \& Technology Education, [s.1.], v. 12, n. 1, p.3-24, 2016.

LISTON, M. The use of video analysis and the Knowledge Quartet in mathematics teacher education programmes. International Journal of Mathematical Education in Science and Technology, [s.1.], v. 46, n. 1, p.1-12, 2015.

MCAULIFFE, S.; LUBBEN, F. Perspectives on pre-service teacher knowledge for teaching early algebra. Perspectives in Education, [s.1.], v. 31, n. 3, p.155-169, 2013.

NEUBRAND, M. Conceptualizations of professional knowledge for teachers of mathematics. ZDM, [s.l], v. 50, n. 4, p. 601-612, 2018.

PETTICREW, M.; ROBERTS, H. Systematic Reviews in the Social Sciences: a practical guide. Oxford: Blackwell Publishing, 2006.

ROWLAND, T. The Knowledge Quartet: the genesis and application of a framework for analysing mathematics teaching and deepening teachers' mathematics knowledge. Sisyphus: Journal of Education, Lisbon, v. 1, n. 3, p.15-43, jan. 2013.

ROWLAND, T.; HUCKSTEP, P.; THWAITES, A. Elementary teachers' mathematics subject knowledge: the Knowledge Quartet and the case of Naomi. Journal of Mathematics Teacher Education, [s.1.], v. 8, n. 3, p.255-281, 2005.

ROWLAND, T.; THWAITES, A.; JARED, L. Triggers of contingency in mathematics teaching. Research in Mathematics Education, [s.1.], v. 17, n. 2, p.74-91, 2015.

ROWLAND, T.; TURNER, F. Developing and using the 'Knowledge Quartet': A framework for the observation of mathematics teaching. The Mathematics Educator, [s.1.], v. 10, n. 1, p.107-123, 2007.

ROWLAND, T.; TURNER, F.; THWAITES, A. Research into teacher knowledge: A stimulus for development in mathematics teacher education practice. ZDM, [s.1.], v. 46, n. 2, p.317-328, 2013. 
SHULMAN, L. S. Those who understand: Knowledge growth in the teaching. Educational Researcher, Washington, v. 15, n. 2, p. 4-14, 1986.

SHULMAN, L. S. Knowledge and teaching: Foundations of the new reform. Harvard Educational Review, Cambridge, v. 57, n. 1, p. 1-22, 1987.

STAHNKE, R.; SCHUELER, S.; ROESKEN-WINTER, B. Teachers' perception, interpretation, and decision-making: a systematic review of empirical mathematics education research. ZDM, [s.l.], v. 48, n. 1-2, p.1-27, 2016.

THWAITES, A.; JARED, L.; ROWLAND, T. Analysing secondary mathematics teaching with the Knowledge Quartet. Research in Mathematics Education, [s.1.], v. 13, n. 2, p.227-228, 2011.

TURNER, F. Using the Knowledge Quartet to develop mathematics content knowledge: the role of reflection on professional development. Research in Mathematics Education, [s.1.], v. 14, n. 3, p.253-271, 2012.

TURNER, F.; ROWLAND, T. The Knowledge Quartet: a means of developing and deepening mathematical knowledge in teaching. In: MATHEMATICAL KNOWLEDGE IN TEACHING SEMINAR SERIES: DEVELOPING AND DEEPENING MATHEMATICAL KNOWLEDGE IN TEACHING, 5, 2008, Loughborough. Anais... Loughborough: Loughborough University, 2008, p. 113.

ÜNVER, S. K. The Knowledge Quartet in the light of the literature on subject matter and pedagogical content knowledge. Acta Didactica Napocensia, [s.1.], v. 11, n. 2, p. 27-42, 2018 a.

ÜNVER, S. K. Views of mathematics student teachers on teacher insights into the teaching process.

International Journal of Instruction, [s.1.], v. 11, n. 4, p. 689-700, 2018 b.

WALSH, R. A case study of pedagogy of mathematics support tutors without a background in mathematics education. International Journal of Mathematical Education in Science and Technology, [s.1.], v. 48, n. 1, p.67-82, 2016.

WESTON, T. L. Using the Knowledge Quartet to quantify mathematical knowledge in teaching: The development of a protocol for initial teacher education. Research in Mathematics Education, [s.l.], v. 15, n. 3, p.286-302, 2013. 